Research Article

\title{
Exploring the Mechanism of Action of Banxia Baizhu Tianma Decoction against Preeclampsia by a Network Pharmacology Approach
}

\author{
Shuqing Cheng ${ }^{1},{ }^{1}$ Xijuan Liu, ${ }^{2}$ Aner Chen, ${ }^{1}$ Haibo Li, ${ }^{3}$ and Lulu Yan ${ }^{3}$ \\ ${ }^{1}$ Department of Obstetrics, Ningbo Women and Children's Hospital, Ningbo 315012, Zhejiang, China \\ ${ }^{2}$ Department of Pediatrics, Ningbo Women and Children's Hospital, Ningbo 315012, Zhejiang, China \\ ${ }^{3}$ The Central Laboratory of Birth Defects Prevention and Control, Ningbo Women and Children's Hospital, Ningbo 315012, \\ Zhejiang, China
}

Correspondence should be addressed to Shuqing Cheng; shuqingc@126.com

Received 24 August 2020; Revised 26 February 2021; Accepted 2 March 2021; Published 12 March 2021

Academic Editor: Letizia Angiolella

Copyright $(2021$ Shuqing Cheng et al. This is an open access article distributed under the Creative Commons Attribution License, which permits unrestricted use, distribution, and reproduction in any medium, provided the original work is properly cited.

\begin{abstract}
Background. Banxia Baizhu Tianma Decoction (BBTD) is a traditional Chinese medicine (TCM) and has been revealed to promote symptoms of preeclampsia (PE) in clinical practice. However, its mechanisms of action and molecular targets for the treatment of $\mathrm{PE}$ are not clear. Method. The potential mechanisms of the BBTD against PE were explored using network pharmacology approach and bioinformatic analysis. The PE animal model was induced by phosphatidylserine/dioleoyl-phosphatidylcholine. The effects of BBTD in the treatment of PE were evaluated in vitro and in vivo. The expressions of RNA and proteins were measured by quantitative real-time polymerase chain reaction and western blotting, respectively. The cell behavior was detected using the MMT assay, Transwell assay, and flow cytometry assay. Results. A total of 173 active compounds of BBTD with 346 targets were identified, and 516 target genes related to PE were also identified from databases. 195 candidate targets for BBTD were screened from the merged PPI network of BBTD-target proteins and PE-related targets. The pathway enrichment analyses showed that the BBTD had the potential to influence a variety of biological pathways. Further pathway-gene network analysis suggested BBTD may improve symptoms of PE via several genes, including MDM2, TP53, RELA, MYC, AKT1, and EGFR. The validation results demonstrated that BBTD treatment promoted pregnancy outcome in the PE animal model. Meanwhile, BBTD regulated the gene expression of MDM2, TP53, RELA, MYC, and EGFR and inhibited the EGFR-JAK/STAT signaling pathway in placental tissue and trophoblast cells. In addition, BBTD promoted the proliferation and invasion and reduced the apoptosis of trophoblast cells. Conclusion. BBTD improved PE by inhibiting the EGFR-JAK/STAT signaling pathway and promoting the proliferation and invasion and reduced the apoptosis of trophoblast cells.
\end{abstract}

\section{Introduction}

Preeclampsia (PE) is a serious pregnancy-related disorder and one of the main causes of maternal and fetal morbidity and mortality worldwide [1]. It is characterized by proteinuria, hypertension, unexplained seizure, and severe headache [2]. Insulin resistance, increased systemic inflammatory response, and endothelial dysfunction are also present in PE [3]. The diagnosis of PE can be made in the absence of proteinuria, if there is laboratory evidence of target organ damage as demonstrated by either thrombocytopenia, renal insufficiency, impaired liver function tests, or pulmonary oedema [4]. PE is a multifactorial disorder that results from changes in complex pathophysiological processes due to several genetic and environmental factors. Diabetes-induced vascular damage, renal disease, pre-existing hypertension, nulliparity, elevated maternal body mass index, and older age are some of the known risk factors of $\mathrm{PE}$. Although our understanding of the pathophysiology of PE has grown enormously over the past few decades, the options for intervention remain limited. Therefore, it is necessary to develop novel therapeutic 
methods for PE, particularly in those developing countries with the greatest burden of disease [5].

Traditional Chinese Medicine (TCM), an important part of complementary and alternative medicine, is widely accepted and used in clinical practice. Banxia Baizhu Tianma Decoction (BBTD) is made up of 13 kinds of herbs: Banxia $10 \mathrm{~g}$ (Arum ternatum), Tianma $15 \mathrm{~g}$ (Rhizoma Gastrodiae), Baishu $15 \mathrm{~g}$ (Atractylodes macrocephala), Fuling $20 \mathrm{~g}$ (Poria cocos), Jupi $12 \mathrm{~g}$ (Citrus reticulata), Cangshu $15 \mathrm{~g}$ (Atractylodes lancea), Gouteng $15 \mathrm{~g}$ (Uncariae Ramulus Cum Uncis), Huangqi 9 g (Hedysarum multijugum), Danshen $15 \mathrm{~g}$ (Radix Salviae), Renshen $9 \mathrm{~g}$ (Panax ginseng), Shichangpu $15 \mathrm{~g}$ (Acoritataninowii Rhizoma), Yujin $15 \mathrm{~g}$ (Curcumae Radix), and Huangbai $6 \mathrm{~g}$ (Phellodendri Chinensis Cortex). It has been reported that the BBTD was effective for many diseases, including vertebrobasilar insufficiency vertigo [6], hyperlipidemia [7], sympathetic nervous cervical spondylosis [8], and hypertension [9]. Recently, the therapeutic function of the BBTD for PE has also been revealed, and it suggested that the BBTD improved the clinical symptoms and related examination indexes of early-onset severe preeclampsia, as well as reducing the incidence of fetal distress and growth restriction [10]. The function and certain mechanism of Gouteng against PE were recently explored; it was suggested that the anti-inflammation effect of Gouteng might be an alternative therapy for PE [11]. However, the comprehensive molecular mechanisms of the BBTD against PE remain unclear.

Network pharmacology with systematic and holistic characteristics has become a promising method to explain the complex interactions between herbs and diseases at the system level. It combines oral bioavailability prediction, multiple drug target prediction, and network analysis to understand the active compounds and therapeutic targets of TCM [12]. It has developed rapidly and been widely used in the TCM studies $[13,14]$. This study provided a systematic method for mapping the mechanism of action of BBTD in the treatment of $\mathrm{PE}$ and identifying potential protein targets that coordinate synergistic effects of TCM. In vitro and in vivo experiments were conducted to validate the effects of BBTD on PE and the underlying mechanisms. We undertook this study to provide insight into the further in-depth development of the basic experimental research and clinical rational application of BBTD.

\section{Materials and Methods}

2.1. Compounds Screening and Targets Identification. To collect the herbal compounds of BBTD, we utilized the Traditional Chinese Medicine Systems Pharmacology Database (TCMSP, http://lsp.nwu.edu.cn/) and the Chinese Academy of Sciences chemistry database (CASC, http://www.organchem.csdb.cn/scdb/main/) to screen active compounds of BBTD. The candidate compounds which have oral bioavailability $(\mathrm{OB}) \geq 30 \%$ and druglikeness (DL) $\geq 0.18$ were identified as active compounds. A bioactive molecule with high $\mathrm{OB}$ displays good DL, which is a qualitative concept utilized in drug design to optimize pharmaceutical and pharmacokinetic properties of molecules, such as chemical stability and solubility. The targets of candidate compounds were obtained from the TCMSP database. The network of compounds-targets was constructed using Cytoscape 3.7.1 software.

2.2. Disease-Related Targets. Details on the human genes associated with PE were acquired from three databases: (1) Comparative Toxicogenomics Database (CTD, https://www. pharmgkb.org/), (2) OMIM (https://www.omim.org/), and (3) GeneCards (https://www.genecards.org/). The gene identifiers were also converted into official gene symbols using an R-script linking the website of PubMed (https:// www.ncbi.nlm.nih.gov/gene/), and only human genes were included for further analysis.

\subsection{Screening of Common Targets and Networks} Construction. Common target genes for drugs and diseases were obtained, and a network of the compound-common targets was constructed. The PPI networks of the compound targets and PE targets were constructed and visualized using Cytoscape 3.7.1 software using the plugin Bisogenet of Cytoscape 3.7.1 software [15], which obtains PPI data from the following six databases: Database of Interacting Proteins, Biological General Repository for Interaction Datasets, Human Protein Reference Database, IntAct Molecular Interaction Database, Molecular Interaction Database, and Biomolecular Interaction Network Database.

2.4. Bioinformatic Analysis. GO and KEGG enrichment analysis was carried out using the Database for Annotation, Visualization, and Integrated Discovery (DAVID, https:// david.ncifcrf.gov, v6.8). Functional categories were enriched within genes $(P<0.05)$ and the top $10 \mathrm{GO}$ functional categories were illustrated. Pathways that had significant changes of $P<0.05$ were identified for further analysis. The genes that significantly regulated pathways were selected for gene-pathway network analysis and key target genes were identified from the gene-pathway network.

2.5. BBTD Preparation. Herbs that consisted of Banxia $10 \mathrm{~g}$, Tianma 15 g, Baishu 15 g, Fuling 20 g, Jupi 12 g, Cangshu 15 g, Gouteng 15 g, Huangqi 9 g, Danshen 15 g, Renshen 9 g, Shichangpu $15 \mathrm{~g}$, Yujin $15 \mathrm{~g}$, and Huangbai $6 \mathrm{~g}$ were decocted for $60 \mathrm{~min}$, and the decoction filtrate was concentrated to $200 \mathrm{~mL}(0.28 \mathrm{~g} / \mathrm{mL})$ to obtain the BBTD decoction.

2.6. Animal Studies. ICR mice were obtained from Kay Biological Technology Co., Ltd. (Shanghai, China). The study was approved by the Research Ethics Committee of Ningbo Women and Children's Hospital. Preeclampsia in mice was induced by tail vein injection of $1 \mathrm{mg}$ phosphatidylserine/dioleoyl-phosphatidylcholine (PS/PC) (SigmaAldrich, St. Louis, MO, USA) suspension from days 5 to 17 of pregnancy as previously described [16]. Saline was used as control. All pregnant female mice were divided into control 
group $(n=8)$ and PE group $(n=32)$. PE mice were orally given BBTD at $3.96,7.92$, and $15.84 \mathrm{~g} / \mathrm{kg} /$ day for 16 days, while sham operation group and the control group were given olive oil for 16 days.

Proteinuria was detected using urine protein test kit (Jiancheng Institute of Biotechnology, Nanjing, China) on day 16. The systolic blood pressure (SBP) of each mice was measured on gestation day 0 (GD0), GD4, GD8, GD12, and GD16 using the tail-cuff method (BP-2000; Visitech Systems, Apex, NC, USA). Then, the mice were anesthetized and sacrificed with $10 \%$ chloral hydrate $(3 \mathrm{~mL} / \mathrm{kg}$, intraperitoneal injection). Placentas and fetuses were harvested from the mice and weighed. The collected placentas were used for subsequent experiments.

2.7. Cell Culture and Treatment. The chorionic trophoblast tissue was isolated from the placentas in the control group and PE group, cut into slices with a thickness of about $3 \mathrm{~mm}$, and digested with $0.25 \%$ pancreatin in a $37^{\circ} \mathrm{C}$ water bath 3 times, each for 10 minutes. Cell suspension was obtained by centrifuging at $3000 \mathrm{r} / \mathrm{min}$ for $5 \mathrm{~min}$ to remove precipitation, washed twice with PBS, then resuspended in DMEM/F12 containing $10 \% \mathrm{FBS}$, and inoculated in a culture plate at $37^{\circ} \mathrm{C}$ and $5 \% \mathrm{CO}^{2}$ in an incubator. After being identified as impurity-free trophoblast cells, they were further cultured in a $37^{\circ} \mathrm{C}, 5 \% \mathrm{CO}^{2}$ incubator. Trophoblast cells from PE mice model pretreated with or without $10 \mathrm{mg} / \mathrm{mL}$ BBTD for $30 \mathrm{~min}$ and trophoblast cells from the control group were used for further experiments.

2.8. MTT Assay. Cell viability was detected using an MTT assay kit (Sigma-Aldrich, St. Louis, MO, USA) according to the manufacturer's protocol. Briefly, trophoblast cells $\left(1 \times 10^{5}\right)$ were seeded into 96-well plates. After culturing for $24,48,72 \mathrm{~h}$, and $96 \mathrm{~h}, 10 \mu \mathrm{L}$ of $5 \mathrm{mg} / \mathrm{mL}$ MTT solution (Shanghai Biyuntian Bio-Technology Co., Ltd., Shanghai, China) was added to each well and then incubated in a $37^{\circ} \mathrm{C}$, $5 \% \mathrm{CO}^{2}$ incubator for $4 \mathrm{~h}$. The absorbance at $570 \mathrm{~nm}$ was measured with a microplate reader (MG LabTech, Durham, NC, USA).

2.9. Flow Cytometry Assay. The effects of BBTD on cell apoptosis were measured using Annexin V-FITC/PI staining kit (Roche, Swiss) according to the manufacturer's instruction. Briefly, the cells were seeded into a 6-well plate for $24 \mathrm{~h}$. Then, the cells were collected and washed with PBS. After centrifugation at $1500 \mathrm{rpm}$ for $3 \mathrm{~min}$, the cell pellet was resuspended in $100 \mu \mathrm{L}$ Annexin V-FITC/PI labeling solution and incubated for $10-15 \mathrm{~min}$. Then, the apoptotic cells were analyzed immediately on a flow cytometer (Beckman Coulter, USA).

2.10. Transwell Assay. Cells were adjusted to $1 \times 10^{5}$ cells $/ \mathrm{mL}$ and $150 \mu \mathrm{L}$ of cell suspension was added into the Transwell chamber (Merck Millipore, Burlington, MA, USA) with a porous membrane pre-coated with or without migration Matrigel solution (Sigma-Aldrich, St. Louis, MO, USA).
Complete medium $(500 \mathrm{~mL})$ was added to the lower chamber. Cells were incubated for $48 \mathrm{~h}$. Cells that had traversed the lower compartment were fixed with methanol, stained with $0.1 \%$ crystal violet (Sigma-Aldrich, St. Louis, MO, USA) and counted by light microscopy.

\subsection{Quantitative Reverse Transcription PCR ( $q R T-P C R)$.} The total RNA was extracted from the cells and placentas tissue using TRIzol (Invitrogen, Carlsbad, CA, USA). cDNA was reverse-transcribed from RNA by iScript advanced cDNA Synthesis Kit (BioRad, Hercules, CA, USA). RT-PCR was performed with SYBR Green chemistry (Thermo Fisher Scientific, MA, USA) on a 7500 fast RT-PCR system. The glyceraldehyde-3-phosphate (GAPDH) mRNA level was used as control in this experiment. All sequences of primers used in RT-PCR are shown in Table 1.

2.12. Western Blot. Total protein was extracted from the cells and placentas tissue by radioimmunoprecipitation assay buffer (Beyotime Biotechnology, Shanghai, China) and stored at $-20^{\circ} \mathrm{C}$. Proteins in each sample were separated by $10 \%$ SDS-PAGE, following gel transfer onto PVDF membranes and blocking in PBS containing 5\% non-fat milk for $2 \mathrm{~h}$ at room temperature. Then, we added primary anti-bodies JAK (cat. No. ab133666, 1:1200, Abcam), p-JAK (cat. No. ab138005, $1: 1200$, Abcam), STAT3 (cat. No. ab68153, 1:1000, Abcam), p-STAT3 (cat. No. ab267373, $1: 1000$, Abcam), EGFR (cat. No. ab52894, 1 : 1000, Abcam), and GAPDH (cat. No. ab8245, 1:1000, Abcam) for $12 \mathrm{~h}$ at $4^{\circ} \mathrm{C}$. The protein was washed with membrane-washing buffer 3 times ( 5 minutes per time), followed by the addition of secondary antibodies and incubation at room temperature for 1 hour. The membrane was visualized using ECL system (Pierce, Rockford, USA), and Image $J$ software was used for band intensity quantification.

2.13. Statistical Analysis. SPSS version 17.0 was used to perform the statistical analysis. All data were presented as mean \pm standard deviation (SD). The differences among the groups were evaluated by one-way ANOVA. $P<0.05$ was considered to be statistically significant.

\section{Results}

3.1. Screening of Active Compounds of BBTD and Their Targets. In total, 245 compounds in BBTD were searched from the TCMSP database. Of these, 9 were from Atractylodes Lancea, 7 were from Atractylodes macrocephala, 4 were from Acoritataninowii Rhizoma, 13 were from Arum ternatum, 15 were from Curcumae Radix, 5 were from Citrus reticulata, 20 were from Hedysarum multijugum, 15 were from Poria cocos, 37 were from Phellodendri Chinensis Cortex, 22 were from Panax ginseng, 65 were from Radix Salviae, and 33 were from Uncariae Ramulus Cum Uncis. Owing to the lack of information about compounds of Rhizoma Gastrodiae in the TCMSP, 13 representative 
TABLE 1: Sequences of primers used in the qPCR.

\begin{tabular}{|c|c|}
\hline Gene name & Sequences $\left(5^{\prime}-3^{\prime}\right)$ \\
\hline \multirow{2}{*}{ TP53 } & Forward: GGCCCACTTCACCGTACTAA \\
\hline & Reverse: GTGGTTTCAAGGCCAGATGT \\
\hline \multirow{2}{*}{ MDM2 } & Forward: GAATCATCGGACTCAGGTACATC \\
\hline & Reverse: TCTGTCTCACTAATTGCTCTCCT \\
\hline \multirow{2}{*}{ RELA } & Forward: CTGCAGTTTGATGATGAAGA \\
\hline & Reverse: TAGGCGAGTTATAGCCTCAG \\
\hline \multirow{2}{*}{ MYC } & Forward: GGCTCCTGGCAAAAGGTCA \\
\hline & Reverse: CTGCGTAGTTGTGCTGATGT \\
\hline \multirow{2}{*}{ AKT1 } & Forward: CCTCCACGACATCGCACTG \\
\hline & Reverse: TCACAAAGAGCCCTCCATTATCA \\
\hline \multirow{2}{*}{ EGFR } & Forward: GCGTCTCTTGCCGGAATGT \\
\hline & Reverse: CTTGGCTCACCCTCCAGAAG \\
\hline \multirow{2}{*}{ PCNA } & Forward: GGCTCTAGCCTGACAAATGC \\
\hline & Reverse: GCCTCCAACACCTTCTTGAG \\
\hline \multirow{2}{*}{ Ki67 } & Forward: AAGCCCTCCAGCTCCTAGTC \\
\hline & Reverse: TCCGAAGCACCACTTCTTCT \\
\hline \multirow{2}{*}{ GAPDH } & Forward: TCGTGGAAGGACTCATGACC \\
\hline & Reverse: AGGGATGATGTTCTGGAGAG \\
\hline
\end{tabular}

compounds of Rhizoma Gastrodiae were obtained from the CASC database. Eventually, 233 candidate compounds were obtained in total after removing the duplicates. 173 were finally selected after removing 60 compounds without any targets (Table S1). Two thousand seven hundred and twentynine targets were identified, and a total of 346 targets of 173 active compounds were collected after removing duplicates.

3.2. Construction of the Compounds-Targets Network. To further understand the complex interactions between the compounds and their corresponding targets at a system level, we constructed a compound-target network based on the candidate compounds of BBTD and their targets (Figure 1). The network contained 519 nods (173 compounds in BBTD and 346 targets) and 2729 edges which indicated the compound-target interactions. All candidate compounds had a median of 12 degrees, suggesting that most compounds of BBTD affected multiple targets. Based on the degree value, the top six compounds were quercetin, kaempferol, luteolin, 7-O-methylisomucronulatol, wogonin, and succinic acid, the degrees of which were $154,63,57,45$, 45 , and 43 , respectively. The $\mathrm{OB}$ of the top six compounds was $46.43,41.88,36.16,74.69,30.68$, and $29.62 \%$, respectively. Therefore, considering the position in the network, they might be the crucial active compounds of BBTD for PE.

3.3. Disease Targets. PE-related targets were identified from GeneCard (218), CTD (310), and OMIM (60) databases. After compensating for data redundancy, 516 target genes were finally included (Table S2). Of these, 106 were targets of the herbs comprising BBTD, which verifies its therapeutic potential as an anti-PE TCM formulation. We constructed and analyzed the compound anti-PE targets network (Figure 2). Based on degree centrality, the top six active compounds in BBTD were quercetin, luteolin, kaempferol, wogonin, naringenin, and tanshinone, the degrees of which were $73,31,28,23,19$, and 17 , respectively.
3.4. Identification of Candidate BBTD Targets Responsible for Treatment of PE. PPI involves various biological processes and is regarded as the primary objective of system biology [17]. In order to reveal the mechanisms of action underlying effects of BBTD on PE, the PPI network of BBTD putative targets and the PPI network of PE-related targets were merged to identify the candidate targets for BBTD against PE. First, a putative target PPI network of BBTD-related genes was constructed with 8687 nodes and 191788 edges on a systems pharmacology platform. After further extraction of PE-related targets, a disease-specific network was constructed with 9401 nodes and 201905 edges. We then merged these two networks to obtain a core PPI network with 7695 nodes and 177332 edges. Subsequently, candidate PE-related proteins modified by BBTD were screened based on the topological features of the core PPI. A node is identified as a significant target if its degree was more than twice the median degree of all nodes in the network [18]; the median degree in our network was 25 . Thus, we constructed a network of significant targets of BBTD with 2039 nodes and 84681 edges. Using a Cytoscape plugin (CytoNCA), we selected the following seven topological features to identify candidate targets: betweenness centrality (BC), degree centrality (DC), closeness centrality (CC), eigenvector centrality (EC), network centrality (NC), and local average connectivity (LAC) [19]. The median values of BC, CC, DC, EC, LAC, and NC were 788.4383, 0.4799, 86, 0.0120, 10.6032, and 11.6740 , respectively. Thus, we identified 195 candidate targets with values for these topological features higher than the median values of CC and two times of other five median values. A representative flowchart of the screening process is presented in Figure 3.

3.5. GO and KEGG Enrichment Analysis. Four hundred ninety-two GO terms, with 316 in biological process, 79 in cellular component, and 97 in molecular function, were significantly enriched $(P$-value $<0.05)$. The top 10 terms are shown in Figure 4 . The highly enriched GO terms in molecular function, cellular component, and biological process included poly(A) binding, protein binding, nucleoplasm, membrane, nucleus, extracellular exosome, telomere organization, nucleosome assembly, and regulation of gene expression. Sixty-two significantly enriched pathways $(P$-value $<0.05)$ including thyroid hormone signaling pathway, estrogen signaling pathway, PI3K-Akt signaling pathway, MAPK signaling pathway, neurotrophin signaling pathway were identified. Top 20 enriched pathways are shown in Figure 5; the size of the spot represented the number of genes and color represented -log FDR value.

3.6. Gene-Pathway Network Analysis. To further investigate the core genes involved in the anti-PE effect of BBTD, the significantly enriched pathways (with $P$-value $<0.001$ ) and the related genes were obtained to construct the genepathway network, as shown in Figure 6. The topological analysis of the 28 pathways and 136 genes was carried out with BC, and the results suggested that RPS6 had the most 


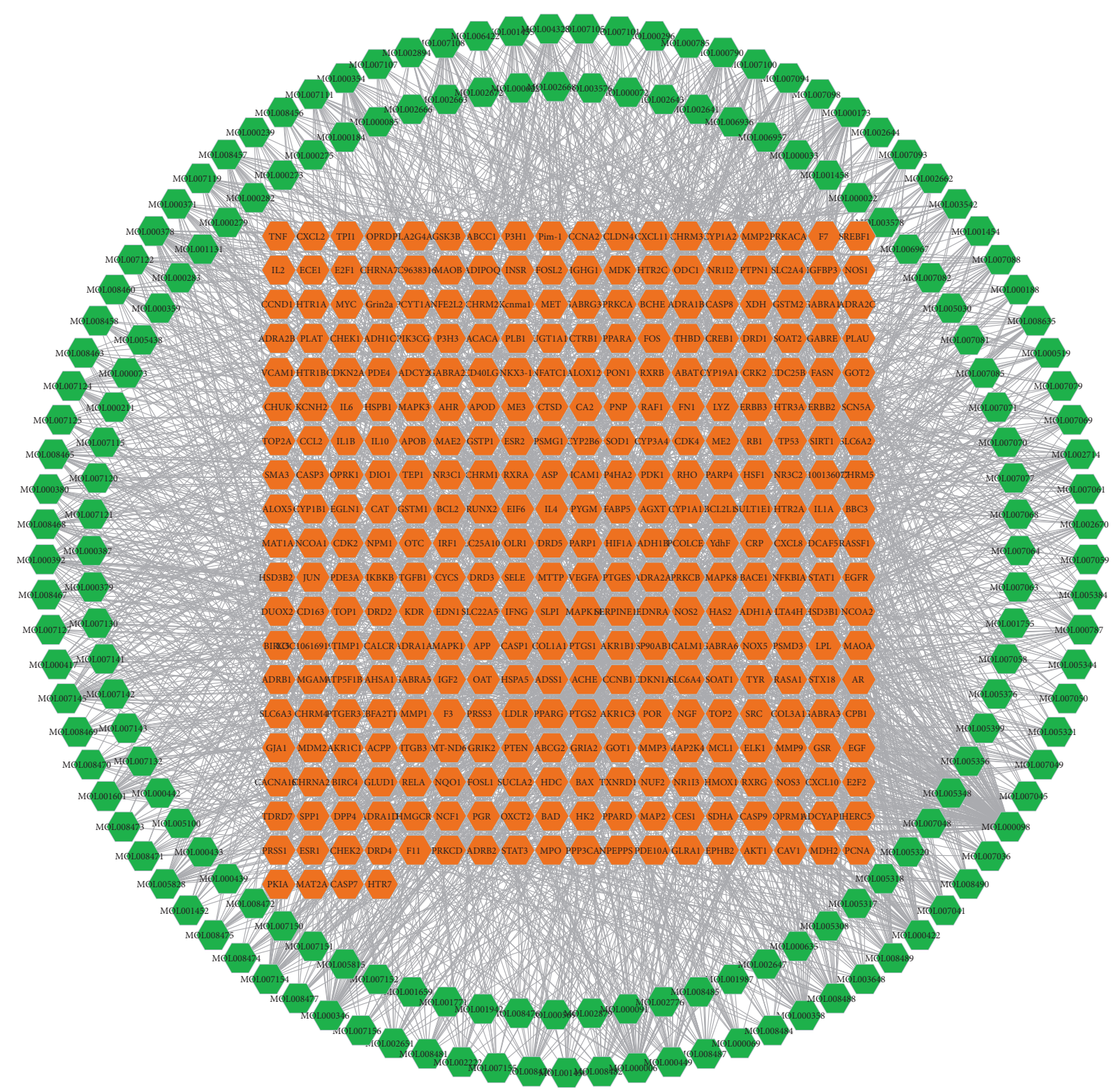

FIGURE 1: Compound-compound targets network of BBTD. The green hexagons represent compounds; the orange hexagons represent the compounds from herbs of BBTD.

maximum BC and was the core target gene. Other several genes also had larger BC, such as MDM2, MAPK1, TP53, NFKB1, RELA, MYC, and DDX5. Among these, MDM2, TP53, RELA, MYC, AKT1, and EGFR were direct targets of BBTD with higher BC, suggesting that they might be the key target genes for BBTD against PE.

3.7. The Effects of BBTD on PE Animal Mice. To study the effect of BBTD on the pregnancy outcome of PE model mice, we compared the systolic blood pressure, proteinuria, and fetal weight of mice in each group. The results showed that BBTD treatment significantly ameliorated SBP and proteinuria levels and increased fetal weights in preeclampsia animal model in a dose-dependent manner (Table 2). Meanwhile, BBTD treatment reduced the elevated gene expression of p53 and EGFR and increased the downregulated gene expression of MDM2, RELA, and MYC in PE animal model (Figure $7(\mathrm{a})$ ). In addition, the upregulated protein expression of EGFR and the elevated ratio of p-JAK/ JAK and p-STAT/STAT in PE mice model were also decreased by BBTD treatment in a dose-dependent manner (Figure 7(b)-7(d)), suggesting that the beneficial effects of BBTD on PE might be associated with the regulation of these core genes and the inhibition of EGFR-JAK/STAT signaling pathway.

3.8. The Effects of BBTD on Trophoblast Cells. We found that the cell proliferation of trophoblast cells was significantly decreased in PE mice model, while BBTD treatment partially reversed this effect (Figure $8(\mathrm{a})$ ). Further, we detected the 


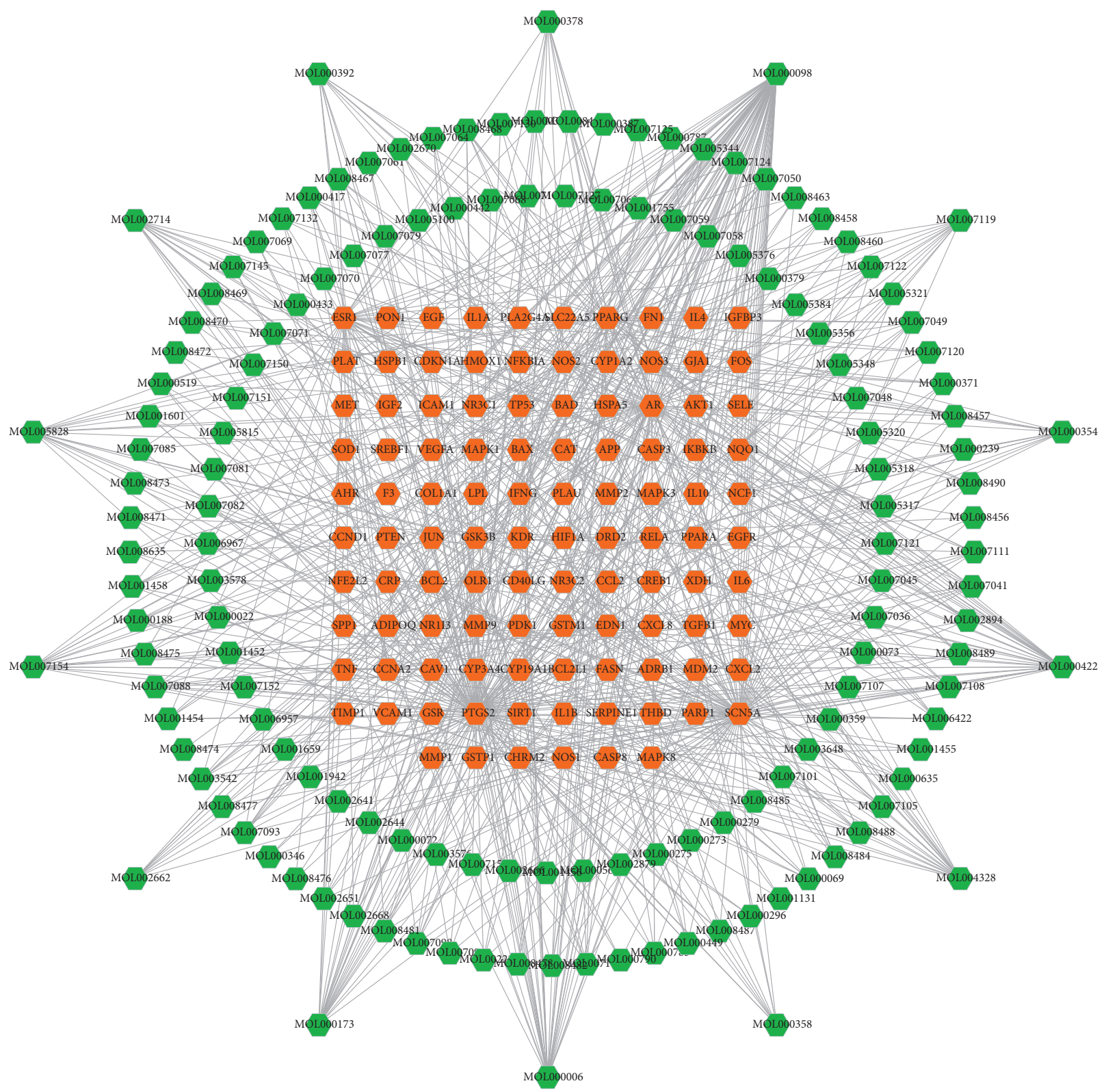

FIgURE 2: Network of compounds-anti-PE targets. The green hexagons represent compounds; the orange hexagons represent the compounds from herbs of BBTD.

mRNA levels of PCNA and Ki67, which indicate the cell proliferation status. Our results showed that the decreased expression of PCNA and Ki67 in PE mice model was upregulated by BBTD treatment (Figure 8(b)). It has been reported that the reduced invasive ability of embryonic trophoblast cells was the initial pathological change in $\mathrm{PE}$ procedure. Our results showed that the number of the invasive trophoblast cells was decreased in the $\mathrm{PE}$ group compared with that in the control group, whereas BBTD treatment significantly increased the number of the invasive trophoblast cells (Figures $8(\mathrm{c})$ and $8(\mathrm{~d})$ ). Flow cytometry assay showed that BBTD treatment significantly reduced the enhanced apoptosis rate of trophoblast cells from PE mice model (Figures 8(e) and 8(f)). In addition, we demonstrated that the decreased expression of MDM2, RELA, and MYC in trophoblast cells from PE mice model was remarkably upregulated by BBTD treatment (Figure $8(\mathrm{~g})$ ). The elevated protein level of EGFR and the ratio of $\mathrm{p}-\mathrm{JAK} / \mathrm{JAK}$ and p-STAT/STAT in trophoblast cells were decreased by BBTD treatment (Figures $8(\mathrm{~h})-8(\mathrm{j})$ ).

\section{Discussion}

In China, the unique theory of TCM has been formed and developed over two thousand years for treating and preventing various diseases, including cardiovascular disease [20], neurological diseases [21], and cancer [22]. The most prominent feature of TCM is the multi-component, multitarget, multi-pathway comprehensive treatment of diseases to achieve the effect of improving symptoms and curing 

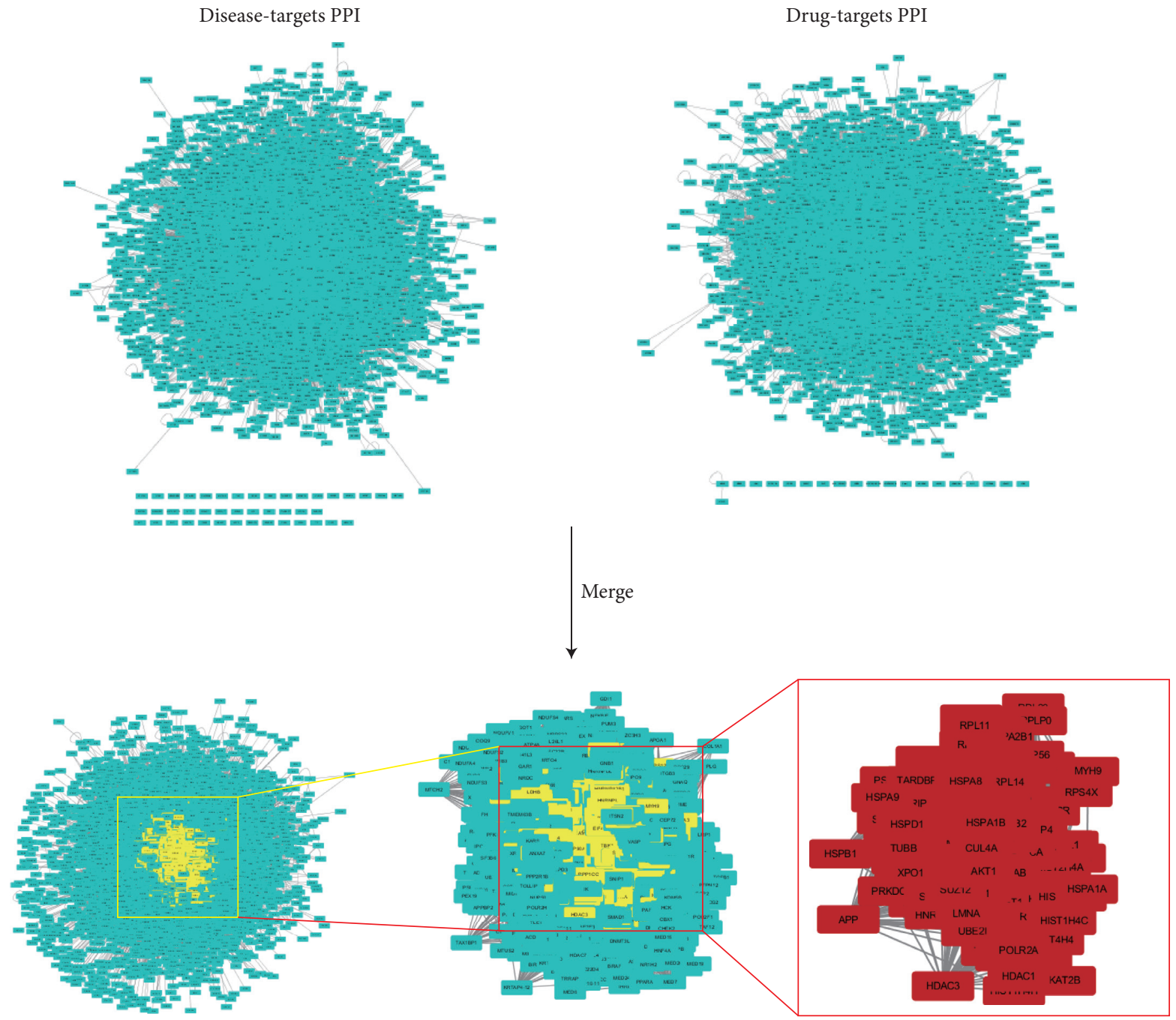

FIgURe 3: PPI network merge and identification of candidate BBTD targets for PE treatment based on topological characteristics.

diseases with fewer side effects. Various active ingredients in complex TCM formulations improve therapeutic effect through synergism [23]. PE is a multifactorial disorder and manifests as a maternal phenotype including hypertension and proteinuria with or without other multiorgan dysfunctions, or as the fetal phenotype including intrauterine growth retardation [24], which is suitable for treatment using TCM theory. The application of TCM or integrated TCM and western medicine on the treatment of PE has attracted widespread attention and research by clinicians. BBTD consists of 13 herbs and is mainly used in the treatment of vertigo [6] and hypertension [25], and recently a study also confirmed its function to improve symptoms of PE [10]. Our data demonstrated that BBTD improved pregnancy outcomes in $\mathrm{PE}$ mice models, including ameliorating SBP and proteinuria levels, as well as increasing fetal weights. Furthermore, the underlying mechanisms of BBTD actions on PE were explored using network pharmacology and validated via in vivo and in vitro experiments.

In the network pharmacology analysis, a compoundcompound targets network of BBTD with 519 nods and
2729 edges was constructed. The results indicated that many compounds with a higher degree, such as quercetin, kaempferol, luteolin, 7-O-methylisomucronulatol, wogonin, and succinic acid, may be the pleiotropically crucial active compounds for BBTD. Quercetin is a polyphenol derived from plants and has been shown to exert multiple pharmacological activities, including anticarcinogenic [26], anti-inflammatory [27], and antiviral activities [28]. It has also been reported that quercetin could be used for the prevention and/or treatment of preeclampsia owing to its protection against the placental secreted messengers released under preeclampsia-like stress conditions like placental hypoxia [29]. Normal pregnancy evokes a systemic inflammatory response and the response is exacerbated in preeclampsia and can account for its clinical features [30]. Kaempferol and wogonins are representative flavonoids with anti-oxidant, anti-inflammatory, anti-cancer, anti-diabetic, anti-osteoarthritic, and immunomodulatory properties [31-33]. Luteolin also showed anti-cancer [34], anti-oxidant, antiinflammatory, and anti-allergic properties [35]. It sug- 


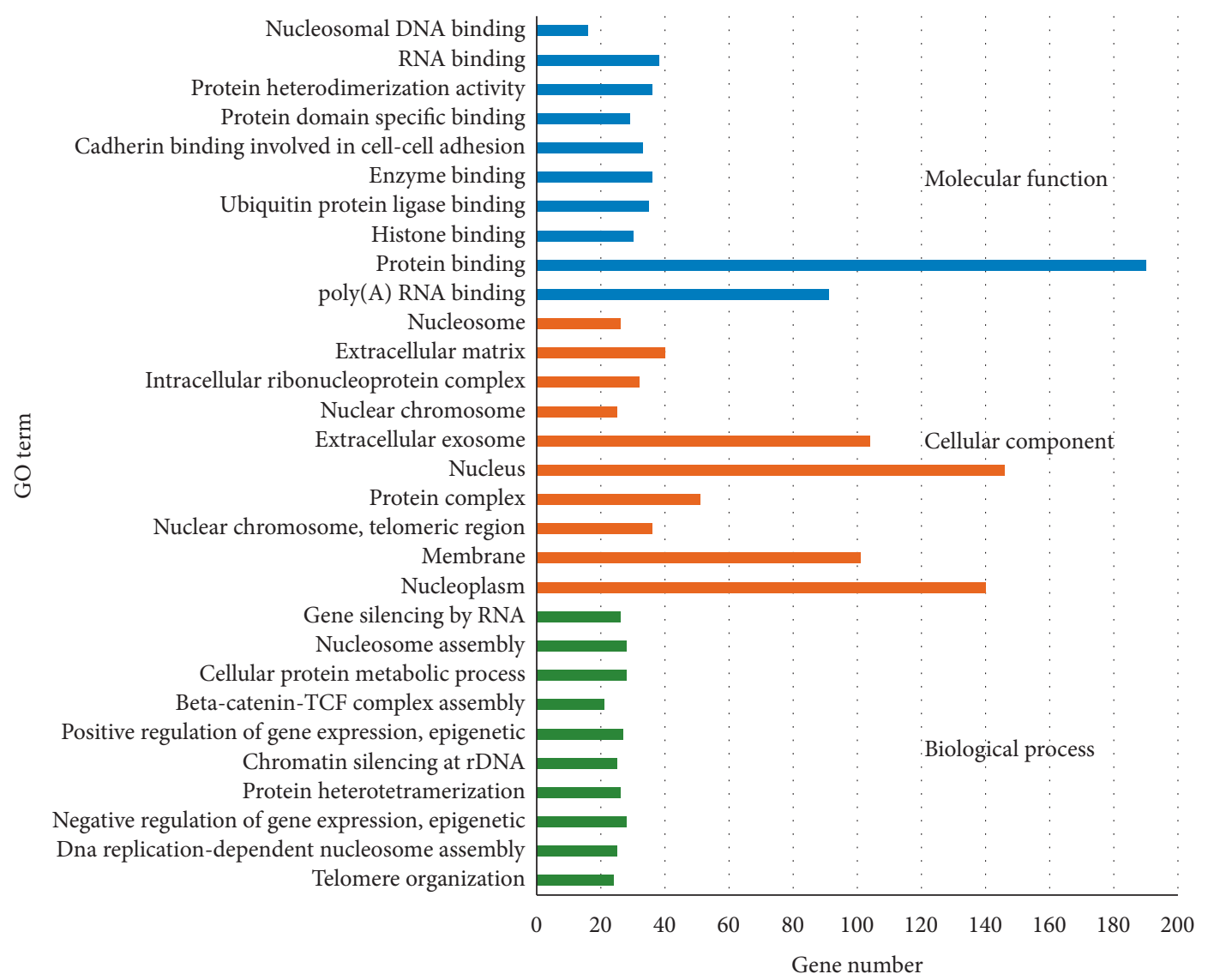

FIGURE 4: Gene ontology terms of candidate targets of BBTD against PE. The top 10 GO functional categories with $P<0.05$ were selected.

gested that kaempferol, luteolin, and wogonins may improve symptoms of PE through their anti-inflammatory and immunomodulatory properties.

To further explore the potential targets of BBTD against PE, we constructed and merged the PPI network of compound-targets and PE-related targets for candidate targets for BBTD against PE. 195 targets were finally identified and used to carry out the enrichment analysis to elucidate the mechanisms underlying the anti-PE effects of BBTD. GO analysis suggested that BBTD regulated some biological processes, such as epigenetic regulation of gene expression, telomere organization, and gene silence. Recent studies demonstrated the importance of maternal epigenetic features, including DNA methylation [36], histone modifications [37], epigenetically regulated miRNA [38], and the effect of imprinted genes on trophoblast growth and invasion in pregnancy [39], which may be potential mechanisms that underlie the theories of disease for PE. Telomeres are essential components of linear chromosomes to protect against chromosome endfusions and genomic instability and are involved in the whole reproductive system. Previous evidence showed that telomeres are shorter in placentas from preeclampsia and telomere aggregate formation in preeclampsia was increased [40]. Therefore, BBTD may exert a regulatory function in the pathogenesis of $\mathrm{PE}$ by regulating telomeres organization. In addition, it may also affect some cellular components and molecular function including nucleoplasm, nucleus, cytosol, protein binding, enzyme binding, and DNA binding in the treatment of PE.

BBTD is a complex TCM formulation with multiple target pathways, which were enriched in KEGG analysis. In the present study, a total of 62 KEGG pathways including thyroid hormone signaling pathway, PI3K-Akt signaling pathway, estrogen signaling pathway, and MAPK signaling pathway were significantly enriched. Thyroid hormones regulate neurodevelopment, probably from early fetal life onwards, and may lead to preterm birth in preeclampsia, and its level is associated with the severity and outcome of preeclampsia [41]. MAPKs participate in the regulation of gene expression, immune response, cell proliferation, apoptosis, and response to oxidative stress, which is one of the mechanisms of immune regulation [42], and the crosstalk between MAPK and PI3K-AKT signaling pathways contributes to the balance of the innate immune responses [43]. Estrogen is produced primarily in the placenta during pregnancy, and its abnormalities could play a crucial role in PE because they are exclusively produced by the placenta and promote angiogenesis and vasodilation [44]. Therefore, BBTD may improve symptoms of PE through the related pathways. In addition, several pathways related to virus were also significantly enriched, such as viral carcinogenesis, hepatitis B, and Epstein-Barr virus infection. It has been reported that viral infection was associated with a twofold 


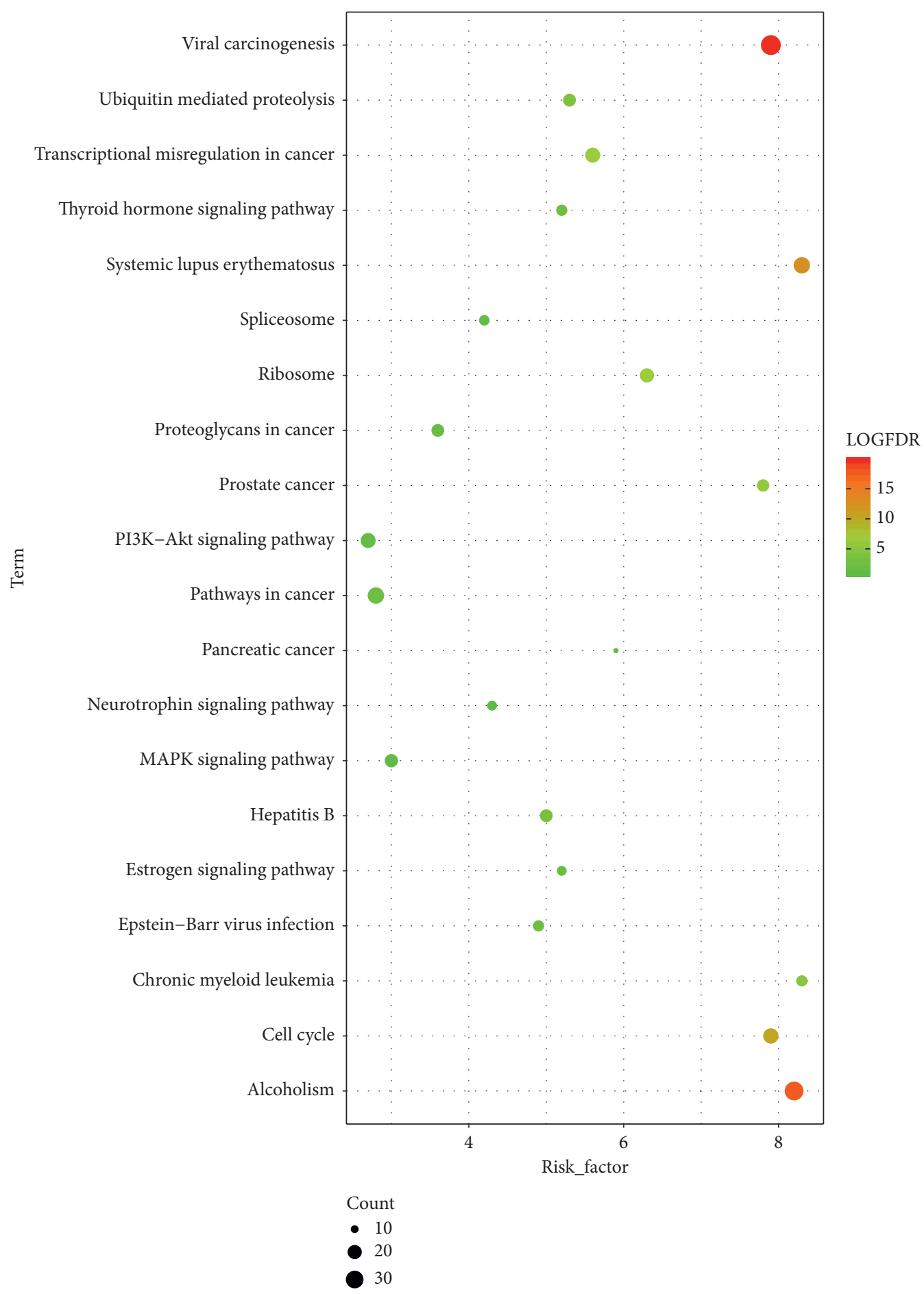

FIGURE 5: KEGG pathway enrichment of candidate targets of BBTD against PE. The top 20 significantly enriched pathways were selected. Size of the spot represents the number of genes and color represents $-\log$ FDR value.

higher risk of preeclampsia [45]. Therefore, the autoimmune response triggered by viral infection might be regulated by BBTD via viral-related pathways. It has also been revealed that dysregulation of canonical cancer molecular pathways occurred in preeclampsia [46], and our results also suggested that BBTD may treat PE via some cancer-related pathways, such as transcriptional misregulation in cancer, pathways in cancer, and prostate cancer pathway. In vitro and in vivo experiments demonstrated that BBTD inhibited the activated EGFR-JAK/STAT signaling pathway in placental tissue and trophoblast cells of PE mice model. EGFR was involved in the PI3K-Akt signaling pathway and MAPK signaling pathway, and the JAK/STAT signaling pathway was associated with the pathogenesis of $\mathrm{PE}[47,48]$.

Gene-pathway network was constructed to investigate the core and key target genes for BBTD against PE. The 


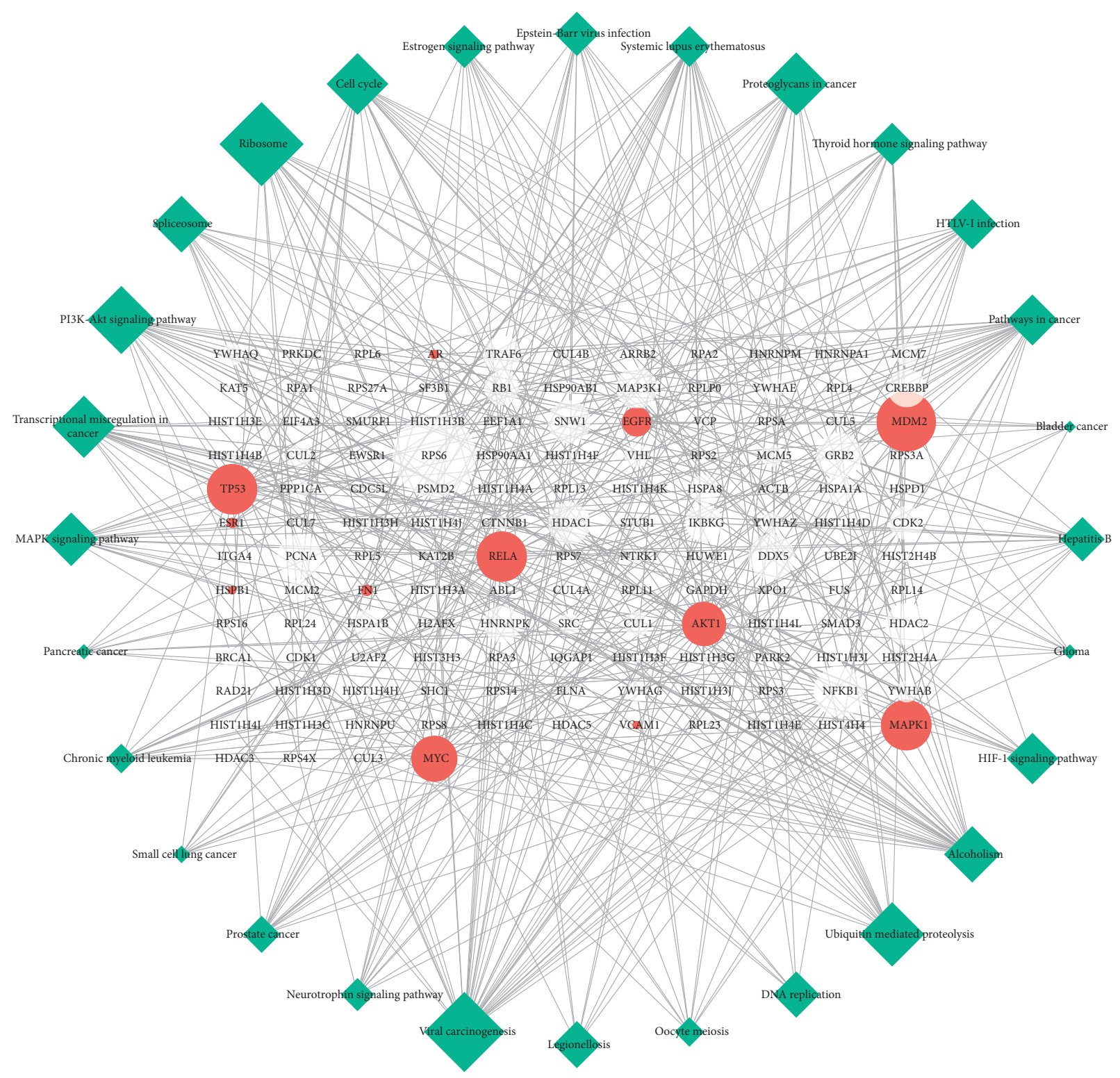

FIGURE 6: Gene-pathway network of BBTD against PE. The topological analysis of 21 pathways and genes was carried out with BC. The blue quadrilaterals represent pathways and the circles represent target genes. Red circles represent direct targets of BBTD. Big size represents larger BC.

TABLE 2: Effects of BBTD on pregnancy outcome of PE mice.

\begin{tabular}{|c|c|c|c|c|c|}
\hline \multirow[t]{2}{*}{ Research indicators } & \multirow[t]{2}{*}{ Control } & \multicolumn{4}{|c|}{$\mathrm{PE}$} \\
\hline & & No treatment & BBTD, $3.96 \mathrm{~g} / \mathrm{kg} /$ day & BBTD, $7.92 \mathrm{~g} / \mathrm{kg} / \mathrm{day}$ & BBTD, $15.84 \mathrm{~g} / \mathrm{kg} /$ day \\
\hline \multicolumn{6}{|l|}{ SBP (mmHg) } \\
\hline GD0 & $110.2 \pm 4.0$ & $111.7 \pm 3.1$ & $110.3 \pm 4.9$ & $107.3 \pm 4.9^{\#}$ & $110.2 \pm 5.2$ \\
\hline GD2 & $104.4 \pm 4.2$ & $111.1 \pm 5.3^{* *}$ & $107.9 \pm 4.1$ & $110.1 \pm 4.2$ & $108.5 \pm 5.0$ \\
\hline GD4 & $107.9 \pm 4.0$ & $123.2 \pm 5.2^{* * *}$ & $119.3 \pm 4.2$ & $118.4 \pm 5.2^{\#}$ & $117.3 \pm 3.5^{\# \#}$ \\
\hline GD8 & $109.1 \pm 3.8$ & $139.7 \pm 3.0^{* * *}$ & $135.6 \pm 2.9^{\#}$ & $129.3 \pm 5.6^{\# \# \#}$ & $124.1 \pm 3.5^{\# \# \#}$ \\
\hline GD16 & $111.8 \pm 5.4$ & $146.9 \pm 4.8^{* * *}$ & $144.8 \pm 3.2$ & $134.6 \pm 4.2^{\# \# \#}$ & $119.5 \pm 3.3^{\# \# \#}$ \\
\hline Proteinuria $(\mathrm{mg} / \mathrm{L})$ & $203.7 \pm 110.5$ & $754.8 \pm 21.9^{* * *}$ & $553.2 \pm 21.0^{\# \# \#}$ & $345.5 \pm 17.0^{\# \# \#}$ & $240.0 \pm 21.9^{\# \# \#}$ \\
\hline Pups weight $(\mathrm{g})$ & $1.46 \pm 0.12$ & $0.72 \pm 0.12^{* * *}$ & $1.15 \pm 0.11^{\# \# \#}$ & $1.28 \pm 0.16^{\# \# \#}$ & $1.32 \pm 0.07^{\# \# \#}$ \\
\hline
\end{tabular}

${ }^{*} P<0.05,{ }^{* *} P<0.01$, and ${ }^{* * *} P<0.001$ vs. control, ${ }^{\#} P<0.05,{ }^{\# \#} \mathrm{P}<0.01$, and ${ }^{\# \# \#} P<0.001$ vs. $\mathrm{PE}$ group. 


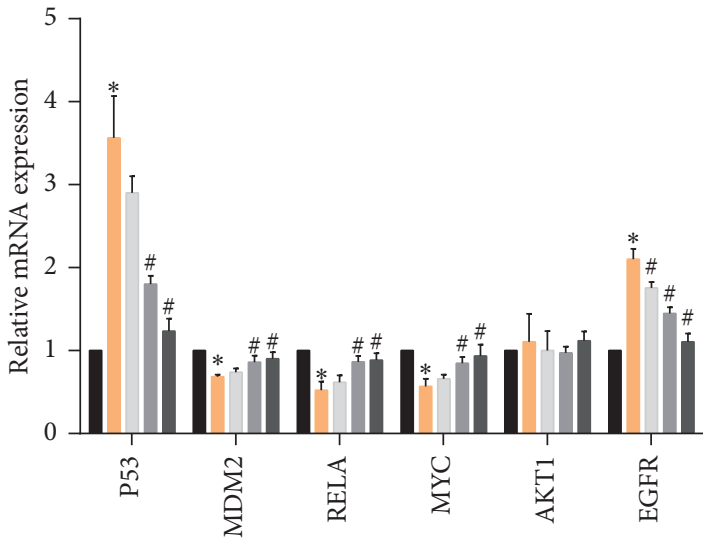

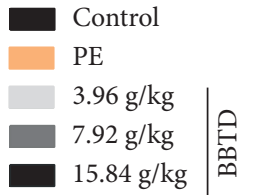

(a)

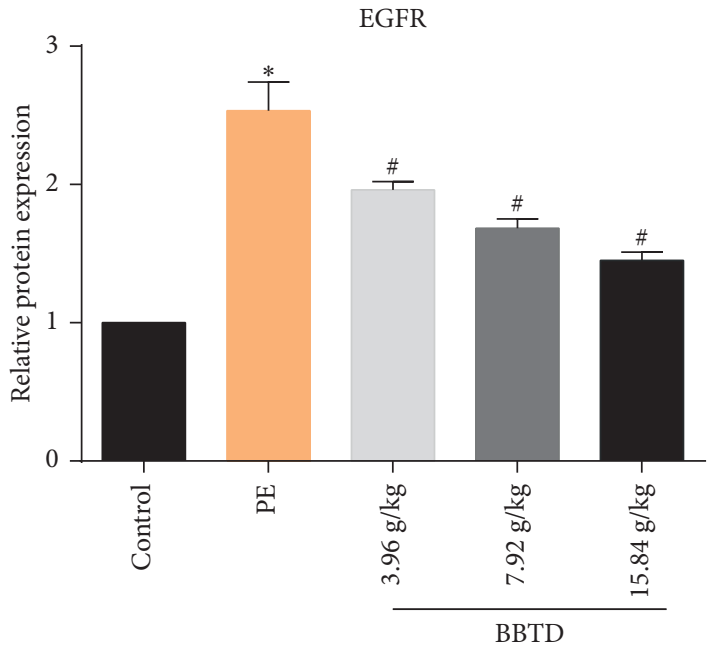

(c)

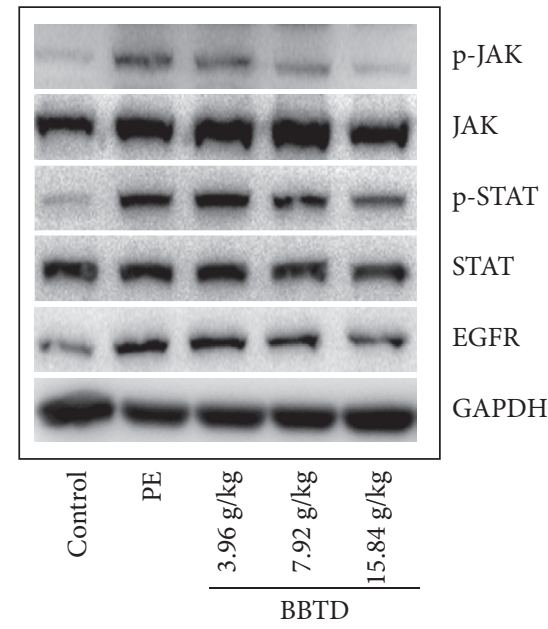

(b)

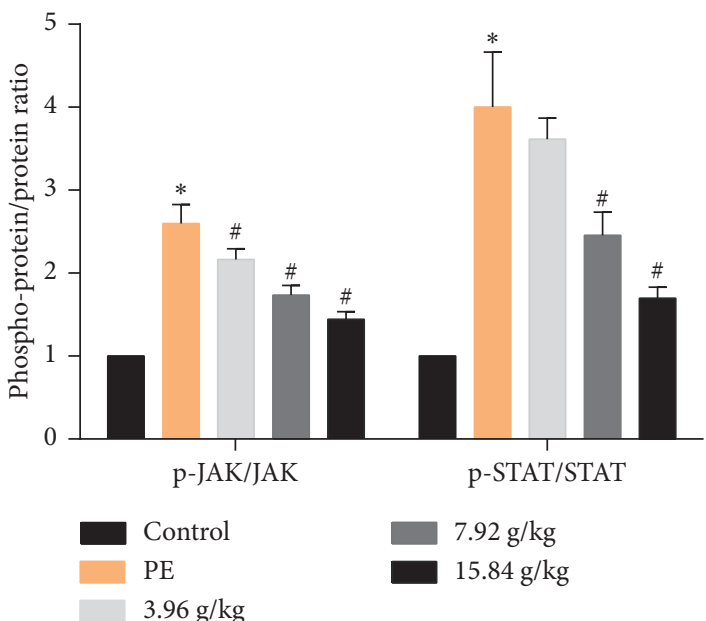

(d)

FIGURE 7: BBTD treatment influenced the gene expression and EGFR-JAK/STAT signaling pathway. (a) The effects of BBTD on the gene expression of P53, MDM2, RELA, MYC, AKT1, and EGFR. (b) Western blot of the EGFR, p-JAK, JAK, p-STAT, and STAT. (c) The statistical analysis of the WB results of EGFR. (d) The statistical analysis of the WB results of p-JAK/JAK and p-STAT/STAT.

results suggested that RPS6 had the maximum BC and it might be the core target and other genes with higher $\mathrm{BC}$, such as MDM2, MAPK1, TP53, NFKB1, RELA, MYC, and DDX5, were identified as key target genes. Moreover, MDM2, TP53, RELA, MYC, AKT1, and EGFR were direct targets genes with higher $\mathrm{BC}$ of $\mathrm{BBTD}$ against $\mathrm{PE}$. In vivo and in vitro experiments confirmed the regulation of p53, MDM2, RELA, MYC, and EGFR gene expression by BBTD. Evidence has confirmed the association between PE and alterations in the p53-pathway, and imbalance in p53 and MDM2 expression promotes excessive apoptosis in villous trophoblast [49]. In addition, it also suggested that p53 may conceivably serve as a potential therapeutic target to improve the placental-related disease and pregnancy outcomes. AKT acts on a variety of targets involved in many cellular processes, such as metabolism, cell cycle, survival, and differentiation [50-52]. It has been demonstrated that placental pathology in preeclampsia and retarded fetuses is related to changes in the expression pattern of EGFR [53], and the antiangiogenic factor, sFlt-1 (soluble FMS-like tyrosine kinase-1), has been strongly implicated in the pathogenesis of preeclampsia and was mediated by EGFR signaling [54]. 


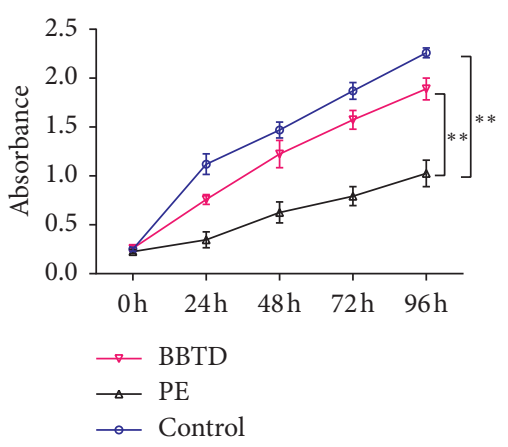

(a)

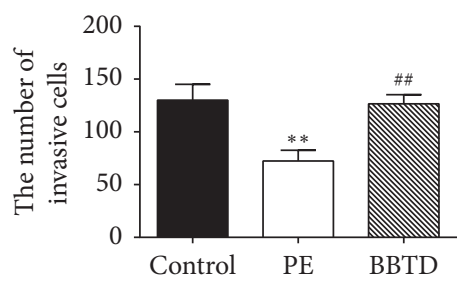

(d)

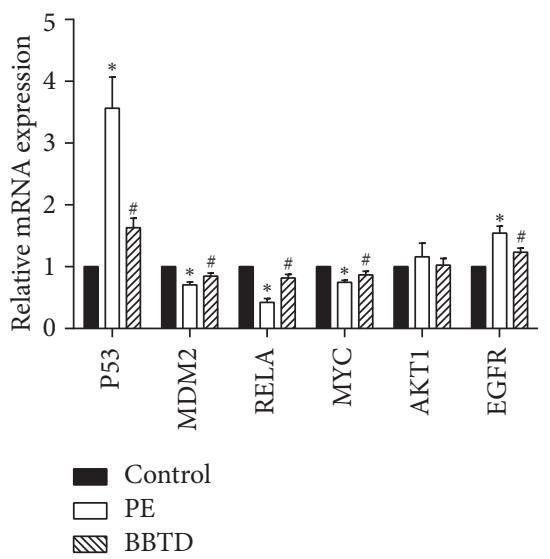

(g)

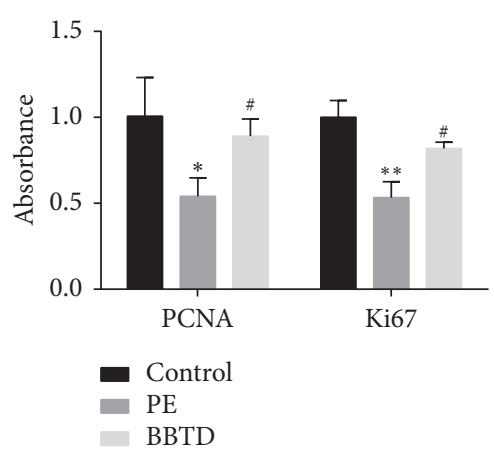

(b)
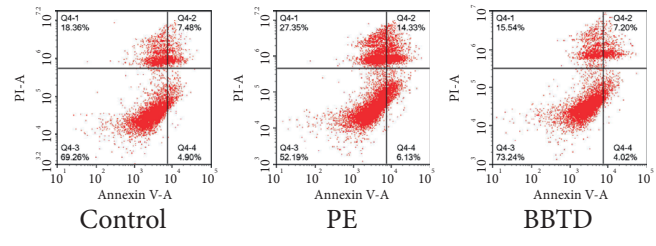

(e)

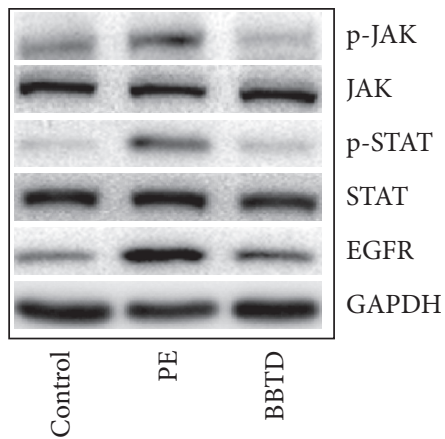

h)

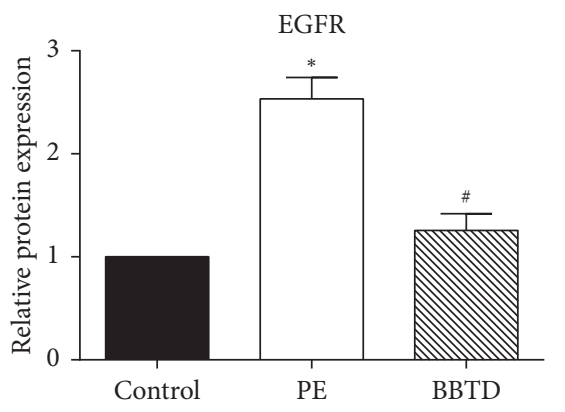

(j)

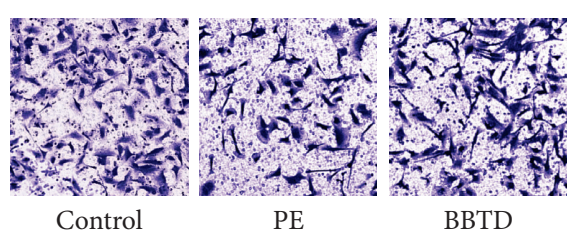

(c)

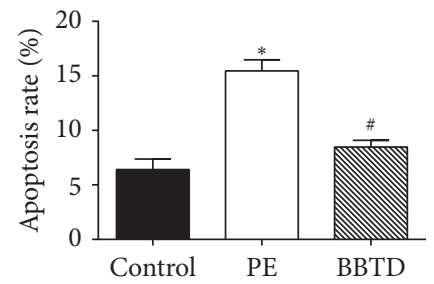

(f)

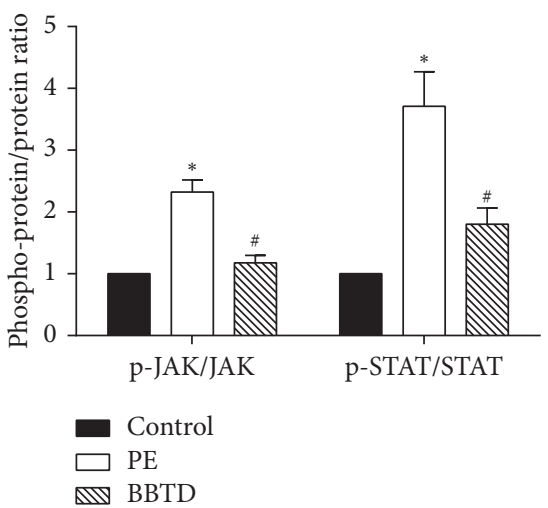

(i)

FIGURE 8: The effects of BBTD on the trophoblast cells. (a) BBTD treatment promoted the proliferation of trophoblast cells, (b) BBTD
treatment upregulated the expression of the indicators of cell proliferation (PCNA and Ki67), (c) BBTD promoted the invasive cell number of trophoblast cells, (d) the statistical analysis of the invasive cell number, (e) BBTD reduced the elevated apoptosis rate of trophoblast cells in PE, (f) the statistical analysis of the apoptosis data, (g) qPCR results in each group, (h) western blot results in each group, (i) the statistical analysis of the p-JAK/JAK and p-STAT/STAT, and $(j)$ the statistical analysis of the EGFR protein expression. 


\section{Conclusion}

In the present study, we applied a network pharmacology approach to predict, elucidate, and confirm the potential mechanisms of the BBTD on PE. It was demonstrated that BBTD promoted pregnancy outcome in PE animal model and the proliferation and invasion and reduced the apoptosis of trophoblast cells. In addition, BBTD functions on the regulation of P53 MDM2, RELA, MYC, and EGFR gene expression and the inhibition of EGFR-JAK/STAT signaling pathway. In this study, we provided the preliminary information and basis for further exploration of BBTD's mechanisms of action on PE, as well as a reference for studying the complex mechanism of action of TCM.

$\begin{array}{ll}\text { Abbreviations } \\ \text { BBTD: } \\ \text { TCM: } & \text { Tranxia Baitional Chinese medicine } \\ \text { PE: } & \text { Preeclampsia } \\ \text { PPI: } & \text { Protein-protein interaction } \\ \text { OB: } & \text { Oral bioavailability } \\ \text { DL: } & \text { Drug-likeness } \\ \text { BC: } & \text { Betweenness centrality } \\ \text { DC: } & \text { Degree centrality } \\ \text { CC: } & \text { Closeness centrality } \\ \text { EC: } & \text { Eigenvector centrality } \\ \text { NC: } & \text { Network centrality } \\ \text { LAC: } & \text { Local average connectivity. }\end{array}$

\section{Data Availability}

The datasets used and/or analyzed during the current study are available from the corresponding author upon reasonable request.

\section{Conflicts of Interest}

The authors declare that they have no conflicts of interest.

\section{Authors' Contributions}

SQC and XJL conceived and designed the study. SQC collected the data. AEC HBL performed animal experiments. SQC, LLY, and XJL conducted the data analysis. SQC and XJL wrote the manuscript. All authors were equally responsible for reviewing the data. All authors read and approved the final manuscript.

\section{Acknowledgments}

This article was supported by the Medical and Health Project of Zhejiang Province (Grant no. 2020KY890) and the Social Development Public Welfare Foundation of Ningbo (Grant nos. 202002N3150 and 2019C50070).

\section{Supplementary Materials}

Table S1: the characteristics of active compounds in BBTD. Table S2: the PE-related targets obtained from different databases. (Supplementary Materials)

\section{References}

[1] P. Gathiram and J. Moodley, "Pre-eclampsia: its pathogenesis and pathophysiolgy," Cardiovascular Journal of Africa, vol. 27, no. 2, pp. 71-78, 2016.

[2] V. A. Rodie, D. J. Freeman, N. Sattar, and I. A. Greer, "Preeclampsia and cardiovascular disease: metabolic syndrome of pregnancy?" Atherosclerosis, vol. 175, no. 2, pp. 189-202, 2004.

[3] E. Lemoine and R. Thadhani, "Affordable preeclampsia therapeutics," Trends in Pharmacological Sciences, vol. 40, no. 2, pp. 85-87, 2019.

[4] Z. Guo, Z. Su, Z. Wang, X. Luo, and R. Lai, "The effect of Chinese herbal medicine Banxia Baizhu Tianma decoction for the treatment of vertebrobasilar insufficiency vertigo: a systematic review and meta-analysis of randomized controlled trials," Complementary Therapies in Medicine, vol. 31, pp. 27-38, 2017.

[5] H. Cai, Y. Guo, Z. Zhao, Y. Chen, S. Zhao, and B. Chen, "Banxia Baizhu Tianma decoction for hyperlipidemia: protocol for a systematic review and meta-analysis," Medicine, vol. 97, no. 44, 2018.

[6] Z. Shengbing, "Curative effect observation of modified Banxia Baizhu Tianma Tang for sympathetic nervous cervical spondylosis," Journal of New Chinese Medicine, vol. 11, no. 5, p. 22, 2017.

[7] C. Huaxing, "The application and value of the Wendan decoction plus the Banxia Baizhu Tianma decoction on hypertension," Clinical Journal of Chinese Medicine, vol. 10, no. 8, p. 20, 2018.

[8] C. Yang, "Clinical observation on treating early onset severe preeclampsia with the Banxia Baizhu Tianma deco," Clinical Journal of Chinese Medicine, vol. 14, no. 11, pp. 9-11, 2019.

[9] L. Wu and X. Xiao, "Evaluation of the effects of Uncaria rhynchophylla alkaloid extract on LPS-induced preeclampsia symptoms and inflammation in a pregnant rat model," Brazilian Journal of Medical and Biological Research, vol. 52, no. 6, 2019.

[10] S. Li and B. Zhang, "Traditional Chinese medicine network pharmacology: theory, methodology and application," Chinese Journal of Natural Medicines, vol. 11, no. 2, pp. 110-120, 2013.

[11] H. Li, L. Zhao, B. Zhang et al., "A network pharmacology approach to determine active compounds and action mechanisms of ge-gen-qin-lian decoction for treatment of type 2 diabetes," Evidence-Based Complementary and Alternative Medicine, vol. 2014, Article ID 495840, 12 pages, 2014.

[12] X. Liang, H. Li, and S. Li, "A novel network pharmacology approach to analyse traditional herbal formulae: the Liu-WeiDi-Huang pill as a case study," Molecular BioSystems, vol. 10, no. 5, pp. 1014-1022, 2014.

[13] A. Martin, M. E. Ochagavia, L. C. Rabasa, J. Miranda, J. Fernandez-de-Cossio, and R. Bringas, "BisoGenet: a new tool for gene network building, visualization and analysis," BMC Bioinformatics, vol. 11, no. 1, p. 91, 2010.

[14] K. Omatsu, T. Kobayashi, Y. Murakami et al., "Phosphatidylserine/phosphatidylcholine microvesicles can induce preeclampsia-like changes in pregnant mice," Seminars in Thrombosis and Hemostasis, vol. 31, no. 3, pp. 314-320, 2005.

[15] V. S. Rao, K. Srinivas, G. N. Sujini, and G. N. S. Kumar, "Protein-protein interaction detection: methods and analysis," International Journal of Proteomics, vol. 2014, pp. 1-12, Article ID 147648, 2014.

[16] Y. Zhang, Z. Li, M. Yang et al., "Identification of GRB2 and GAB1 coexpression as an unfavorable prognostic factor for 
hepatocellular carcinoma by a combination of expression profile and network analysis," PLoS One, vol. 8, no. 12, Article ID e85170, 2013.

[17] Y. Jiang, N. Liu, S. Zhu, X. Hu, D. Chang, and J. Liu, "Elucidation of the mechanisms and molecular targets of Yiqi Shexue formula for treatment of primary immune thrombocytopenia based on network pharmacology," Frontiers in Pharmacology, vol. 10, p. 1136, 2019.

[18] P. Hao, F. Jiang, J. Cheng, L. Ma, Y. Zhang, and Y. Zhao, "Traditional Chinese medicine for cardiovascular disease," Journal of the American College of Cardiology, vol. 69, no. 24, pp. 2952-2966, 2017.

[19] Z. Ren and P. Zuo, "Neural regeneration: role of traditional Chinese medicine in neurological diseases treatment," Journal of Pharmacological Sciences, vol. 120, no. 3, pp. 139-145, 2012.

[20] J. Nie, C. Zhao, L. Deng et al., "Efficacy of traditional Chinese medicine in treating cancer," Biomedical Reports, vol. 4, no. 1, pp. 3-14, 2016.

[21] Y. Li, H. Zhou, J. Xie et al., "A novel method for evaluating the cardiotoxicity of traditional Chinese medicine compatibility by using support vector machine model combined with metabonomics," Evidence-based Complementary and Alternative Medicine, vol. 2016, Article ID 6012761, 7 pages, 2016.

[22] R. B. Ness and J. M. Roberts, "Heterogeneous causes constituting the single syndrome of preeclampsia: a hypothesis and its implications," American Journal of Obstetrics and Gynecology, vol. 175, no. 5, pp. 1365-1370, 1996.

[23] C. S. Tan, Y. C. Loh, C. H. Ng et al., "Anti-hypertensive and vasodilatory effects of amended Banxia Baizhu Tianma Tang," Biomedicine \& Pharmacotherapy, vol. 97, pp. 985-994, 2018.

[24] P. Nair, A. Malhotra, and D. K. Dhawan, "Curcumin and quercetin trigger apoptosis during benzo (a) pyrene-induced lung carcinogenesis," Molecular and Cellular Biochemistry, vol. 400, no. 1-2, pp. 51-56, 2015.

[25] M. Lesjak, I. Beara, N. Simin et al., "Antioxidant and antiinflammatory activities of quercetin and its derivatives," Journal of Functional Foods, vol. 40, pp. 68-75, 2018.

[26] W. Wu, R. Li, X. Li et al., "Quercetin as an antiviral agent inhibits influenza A virus (IAV) entry," Viruses, vol. 8, no. 1, p. 6, 2016.

[27] D. Pellaers, P. Vangrieken, P. Schiffers, S. Al-Nasiry, and F.-J. Schooten, “310. Quercetin protects against the vascular contractile effect of placental secreted messengers released under placental hypoxia: an in-vitro model of preeclampsia," Pregnancy Hypertension, vol. 13, pp. S121-S122, 2018.

[28] C. W. Redman and I. L. Sargent, "Preeclampsia and the systemic inflammatory response," Seminars in Nephrology, vol. 24, no. 6, pp. 565-570, 2004.

[29] M.-S. Tsai, Y.-H. Wang, Y.-Y. Lai et al., "Kaempferol protects against propacetamol-induced acute liver injury through CYP2E1 inactivation, UGT1A1 activation, and attenuation of oxidative stress, inflammation and apoptosis in mice," Toxicology Letters, vol. 290, pp. 97-109, 2018.

[30] S. Khan, D. Zhang, Y. Zhang, M. Li, and C. Wang, "Wogonin attenuates diabetic cardiomyopathy through its anti-inflammatory and anti-oxidative properties," Molecular and Cellular Endocrinology, vol. 428, pp. 101-108, 2016.

[31] N. Sharma, A. K. Singh, S. S. Sodhi et al., "Anti-tumor activity of wogonin, an extract from Scutellaria baicalensis, through regulating different signaling pathways," Chinese Journal of Natural Medicines, vol. 15, no. 1, pp. 15-40, 2017.

[32] Y. Lin, R. Shi, X. Wang, and H.-M. Shen, "Luteolin, a flavonoid with potential for cancer prevention and therapy," Current Cancer Drug Targets, vol. 8, no. 7, pp. 634-646, 2008.
[33] G. Seelinger, I. Merfort, and C. Schempp, “Anti-oxidant, antiinflammatory and anti-allergic activities of luteolin," Planta Medica, vol. 74, no. 14, pp. 1667-1677, 2008.

[34] R. K. Yuen, M. S. Peñaherrera, P. Von Dadelszen, D. E. McFadden, and W. P. Robinson, "DNA methylation profiling of human placentas reveals promoter hypomethylation of multiple genes in early-onset preeclampsia," European Journal of Human Genetics, vol. 18, no. 9, pp. 1006-1012, 2010.

[35] C. Munaut, L. Tebache, S. Blacher, A. Noël, M. Nisolle, and F. Chantraine, "Dysregulated circulating miRNAs in preeclampsia," Biomedical Reports, vol. 5, no. 6, pp. 686-692, 2016.

[36] Y. Nomura, R. M. John, A. B. Janssen et al., "Neurodevelopmental consequences in offspring of mothers with preeclampsia during pregnancy: underlying biological mechanism via imprinting genes," Archives of Gynecology and Obstetrics, vol. 295, no. 6, pp. 1319-1329, 2017.

[37] T. Biron-Shental, R. Sukenik-Halevy, Y. Sharon et al., "Short telomeres may play a role in placental dysfunction in preeclampsia and intrauterine growth restriction," American Journal of Obstetrics and Gynecology, vol. 202, no. 4, p. 381, 2010.

[38] Y. Li, T. Meng, N. Hao et al., "Immune regulation mechanism of Astragaloside IV on RAW264.7 cells through activating the NF- $\kappa \mathrm{B} / \mathrm{MAPK}$ signaling pathway," International Immunopharmacology, vol. 49, pp. 38-49, 2017.

[39] M. G. Rohani, D. H. DiJulio, J. Y. An, B. M. Hacker, B. A. Dale, and W. O. Chung, "PAR1- and PAR2-induced innate immune markers are negatively regulated by $\mathrm{PI} 3 \mathrm{~K} / \mathrm{Akt}$ signaling pathway in oral keratinocytes," BMC Immunology, vol. 11, no. 1, p. 53, 2010.

[40] N. Berkane, P. Liere, J.-P. Oudinet et al., "From pregnancy to preeclampsia: a key role for estrogens," Endocrine Reviews, vol. 38, no. 2, pp. 123-144, 2017.

[41] R. Moslehi, J. L. Mills, C. Signore, A. Kumar, X. Ambroggio, and A. Dzutsev, "Integrative transcriptome analysis reveals dysregulation of canonical cancer molecular pathways in placenta leading to preeclampsia," Scientific Reports, vol. 3, p. 2407, 2013.

[42] A. N. Sharp, A. E. Heazell, D. Baczyk et al., "Preeclampsia is associated with alterations in the p53-pathway in villous trophoblast," PLoS One, vol. 9, no. 1, Article ID e87621, 2014.

[43] N. Milchev, I. Batashki, D. Staribratova, and Z. Zaprianov, "Trophoblast expression of EGFR (epidermal growth factor receptor) in the preeclampsia placenta," Akusherstvo I Ginekologiia, vol. 45, no. 2, pp. 21-24, 2006.

[44] N. Varnier, M. A. Brown, M. Reynolds et al., "Indications for delivery in pre-eclampsia," Pregnancy Hypertension, vol. 11, pp. 12-17, 2018.

[45] L. A. Magee, L. Kenny, S. Ananth Karumanchi, F. McCarthy, S. Saito, and D. R. Hall, "The hypertensive disorders of pregnancy: ISSHP classification, diagnosis and management recommendations for international practice 2018," Pregnancy Hypertension, vol. 72, 2018.

[46] S. Alahari, M. Post, A. Rolfo, R. Weksberg, and I. Caniggia, "Compromised JMJD6 histone demethylase activity affects VHL gene repression in preeclampsia," The Journal of Clinical Endocrinology \& Metabolism, vol. 103, no. 4, pp. 1545-1557, 2018.

[47] D. Sardana, S. Nanda, and S. Kharb, "Thyroid hormones in pregnancy and preeclampsia," Journal of the Turkish German Gynecological Association, vol. 10, no. 3, pp. 168-171, 2009. 
[48] L. O. Rustveld, S. F. Kelsey, and R. Sharma, "Association between maternal infections and preeclampsia: a systematic review of epidemiologic studies," Maternal and Child Health Journal, vol. 12, no. 2, pp. 223-242, 2008.

[49] Z. M. Zhao and J. Jiang, "Lowly expressed EGFR-AS1 promotes the progression of preeclampsia by inhibiting the EGFR-JAK/STAT signaling pathway," European Review for Medical and Pharmacological Sciences, vol. 22, no. 19, pp. 6190-6197, 2018.

[50] H. Qu, Q. Yu, B. Jia, W. Zhou, Y. Zhang, and L. Mu, "HIF-3 $\alpha$ affects preeclampsia development by regulating EVT growth via activation of the Flt-1/JAK/STAT signaling pathway in hypoxia," Molecular Medicine Reports, vol. 23, no. 1, 2021.

[51] D. P. Brazil and B. A. Hemmings, "Ten years of protein kinase B signalling: a hard Akt to follow," Trends in Biochemical Sciences, vol. 26, no. 11, pp. 657-664, 2001.

[52] J. A. Engelman, J. Luo, and L. C. Cantley, "The evolution of phosphatidylinositol 3-kinases as regulators of growth and metabolism," Nature Reviews Genetics, vol. 7, no. 8, pp. 606-619, 2006.

[53] E. Gonzalez and T. E. McGraw, "The Akt kinases: isoform specificity in metabolism and cancer," Cell Cycle, vol. 8, no. 16, pp. 2502-2508, 2009.

[54] R. Hastie, F. C. Brownfoot, N. Pritchard et al., "EGFR (epidermal growth factor receptor) signaling and the mitochondria regulate sFlt-1 (soluble FMS-like tyrosine kinase-1) secretion," Hypertension, vol. 73, no. 3, pp. 659-670, 2019. 